\title{
Young cancer researchers rewarded
}

$\mathrm{M}$ emorial Sloan-Kettering Cancer Center (MSKCC) recently awarded its Paul Marks Prize for Cancer Research to Arul Chinnaiyan, at the University of Michigan; Matthew Meyerson, at the Dana Farber Cancer Institute; and David Sabatini, at the Whitehead Institute. When announcing the news, MSKCC President Harold Varmus noted that, "Each of the researchers we are honoring has already built a body of work that has advanced the field of cancer research" (1).

The Paul Marks Prize for Cancer Research has been biennially awarded to scientists under the age of 46 since its inception in 2001. Named for Paul A. Marks, President Emeritus of Memorial Sloan-Kettering and president and chief executive officer of MSKCC between 1980 and 1999, the prize was created upon his retirement to honor his many contributions to the center.

This year's recipients will each receive $\$ 50,000$ and speak about their work at an MSKCC public symposium to be held at the start of December. They were selected by a committee of cancer research experts chaired by Titia de Lange, a winner of the inaugural Paul Marks Prize and a Rockefeller University professor.

In selecting the winners, committee chairman de Lange was keen to point out that, "Although all three winners are focused primarily on working in the laboratory, the translational aspect of their discoveries has already begun to

\section{"Each of the researchers we are honoring has already built a body of work that has advanced the field of cancer research."}

influence the treatments that cancer patients receive" (1). Chinnaiyan is being recognized predominantly for his work identifying that chromosomal rearrangements that result in gene fusion of the 5 -untranslated region of the androgenregulated gene transmembrane protease, serine 2 (TMPRSS2) with the genes encoding any of 3 ETS transcription factor family members (ERG, ETV1, or ETV4) are detected in more than half of all prostate cancers $(2,3)$. These fusion genes mainly generate truncated ETS proteins - rather than chimeric proteins - that are believed to drive prostate cancer development. Given the prevalence of prostate cancer (it accounts for approximately one-third of all cancer diagnoses in American men), researchers are seeking to determine whether TMPRSS2:ETS fusion genes provide an early detection diagnostic marker or a marker of aggressive disease. Others, including Chinnaiyan, are seeking to develop small-molecule inhibitors of the protein products of the TMPRSS2:ETS fusion genes in the hope that these will provide targeted prostate cancer therapeutics in the same way that imatinib, which inhibits the protein product of the $B C R: A B L$ oncogenic fusion gene responsible for chronic myeloid leukemia (CML), is a targeted therapy for CML.

Meyerson is being rewarded mainly for his work defining both the role of EGFR mutations in driving lung cancer and how these mutations determine a patient's responsiveness to treatment with smallmolecule EGFR tyrosine kinase inhibitors such as erlotinib and gefitinib $(4,5)$. In 2003, the FDA approved gefitinib as a therapy for non-small cell lung cancer, despite the fact that only a small proportion of patients with the disease responded well to treatment and no one knew why. Two independent research groups, one headed by Meyerson, provided an answer to this conundrum in 2004: response to treatment with gefitinib correlated with the presence in the tumor tissue of somatic mutations in the EGFR gene $(4,6)$. These observations provided the underlying rationale for screening patients diagnosed with lung cancer for the presence of EGFR mutations, which is now standard in many clinics, in order to determine the most appropriate therapeutic intervention. Meyerson is now working on identifying and understanding other genetic alterations that drive lung cancer with the hope that additional personalized therapies can be developed for this disease, the most common cause of cancer-related death in both men and women.

As a graduate student, Sabatini identified mammalian target of rapamycin (mTOR; ref. 7), inhibitors of which are now approved for the treatment of advanced renal cell carcinoma. Sabatini told the JCI that he "was drawn to studying the mechanism of action of rapamycin because it was clear back then that, whatever its target was, it would be interesting." Ever since, he has worked on dissecting the molecular

\section{"The translational aspect of [the winners'] discoveries has already begun to influence the treatments that cancer patients receive."}

components of the TOR pathway because he "realized that identifying mTOR was only the tip of what there was to learn." Sabatini noted to the JCI that he was told by many to study something other than mTOR, but he is glad he stuck with it. His dedication to understanding how dysregulation of this pathway contributes to the growth of cancer cells and the pioneering techniques that he developed to study it are key reasons why he is being recognized by the MSKCC. As the mTOR pathway is regulated by nutrients, and therefore metabolism, Sabatini told the JCI that he is very excited about receiving the Paul Marks Prize because he feels that it is "a recognition of the increasing awareness of the importance of metabolic and nutrient signaling in tumorigenesis."

\section{Karen Honey}

1. Memorial Sloan-Kettering Cancer Center. Paul Marks Prize recognizes three young cancer researchers. http://www.mskcc.org/mskcc/html/94886. cfm. Published September 29, 2009. Accessed November 3, 2009.

2. Tomlins, S.A., et al. 2005. Recurrent fusion of TMPRSS2 and ETS transcription factor genes in prostate cancer. Science. 310:644-648.

3. Tomlins, S.A., et al. 2006. TMPRSS2:ETV4 gene fusions define a third molecular subtype of prostate cancer. Cancer Res. 66:3396-3400.

4. Paez, J.G., et al. 2004. EGFR mutations in lung cancer: correlation with clinical response to gefitinib therapy. Science. 304:1497-1500.

5. Greulich, H., et al. 2005. Oncogenic transformation by inhibitor-sensitive and -resistant EGFR mutants. PLoS Med. 2:e313.

6. Lynch, T.J., et al. 2004. Activating mutations in the epidermal growth factor receptor underlying responsiveness of non-small-cell lung cancer to Gefitinib. N. Engl. J. Med. 350:2129-2139.

7. Sabatini, D.M., Erdjument-Bromage, H., Lui, M., Tempst, P., and Snyder, S.H. 1994. RAFT1: a mammalian protein that binds to FKBP12 in a rapamycin-dependent fashion and is homologous to yeast TORs. Cell. 78:35-43. 